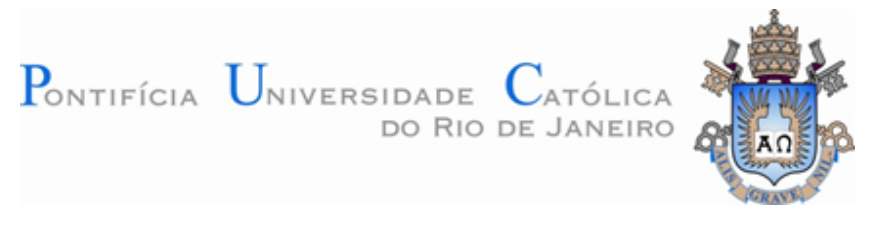

Andréa Viviane de Oliveira Costa

\title{
Indicadores de sustentabilidade para instituições de ensino superior: contribuições para a Agenda Ambiental PUC-Rio
}

Dissertação apresentada ao Programa de Pósgraduação em Metrologia da PUC-Rio como requisito parcial para obtenção do título de Mestre em Metrologia (Área de concentração: Metrologia para Qualidade e Inovação).

Orientadores: Prof ${ }^{a}$ Maria Fatima Ludovico de Almeida Prof. Prof. Fabrício Casarejos 
Andréa Viviane de Oliveira Costa

\begin{abstract}
Indicadores de sustentabilidade para instituições de ensino superior: contribuições para a Agenda Ambiental PUC-Rio
\end{abstract}

Dissertação apresentada como requisito parcial para obtenção do grau de Mestre pelo Programa de Pós-Graduação em Metrologia da PUC-Rio. Aprovada pela Comissão Examinadora abaixo assinada

Prof ${ }^{a}$ Maria Fatima Ludovico de Almeida

Orientadora

Programa de Pós-Graduação em Metrologia - PUC-Rio

Prof. Fabrício Casarejos

Co-orientador

Programa de Pós-Graduação em Metrologia - PUC-Rio

Prof. Maurício Nogueira Frota

Programa de Pós-Graduação em Metrologia - PUC-Rio

Prof. Luis Felipe Guanaes Rego Núcleo Interdisciplinar de Meio Ambiente - PUC-Rio

Prof. Osvaldo Luiz Gonçalves Quelhas

Universidade Federal Fluminense

Prof. José Eugenio Leal Coordenador Setorial do Centro Técnico Científico - PUC-Rio 
Todos os direitos reservados. É proibida a reprodução total ou parcial do trabalho sem autorização da universidade, do autor e de seus orientadores.

\section{Andréa Viviane de Oliveira Costa}

Graduou-se em Engenharia Civil - Habilitação Cartografia pela Universidade do Estado do Rio de Janeiro (UERJ) em 1992. Possui Licenciatura Plena em Física e Matemática. Bolsista do CNPq no Programa de Pós-graduação em Metrologia, Qualidade e Inovação (PósMQI).

Ficha Catalográfica

Costa, Andréa Viviane de Oliveira

Indicadores de sustentabilidade para instituições de ensino superior: contribuições para a Agenda Ambiental PUC-Rio / Andréa Viviane de Oliveira Costa; orientadores: Maria Fatima Ludovico de Almeida. Casarejos, Fabrício - 2012.

126 f. : il. (color.) ; $30 \mathrm{~cm}$

Dissertação (mestrado)-Pontifícia Universidade Católica do Rio de Janeiro, Centro Técnico Científico, 2012.

Inclui bibliografia e anexos

1. Metrologia - Teses. 2. Sustentabilidade. 3. Desenvolvimento sustentável. 4. Instituições de ensino superior. 5. IES. 6. Campus sustentável. 7. Indicadores de sustentabilidade. 8. Agenda Ambiental PUC-Rio. I. Almeida, Maria Fatima Ludovico de. II. Casarejos, Fabrício. III. Pontifícia Universidade Católica do Rio de Janeiro. Programa de PósGraduação em Metrologia para Qualidade e Inovação. IV. Título. 


\section{Agradecimentos}

Para a realização deste trabalho contribuíram várias pessoas, às quais não poderia deixar de agradecer.

Em primeiro lugar, gostaria de apresentar os meus sinceros agradecimentos aos meus orientadores, à Professora Maria Fatima Ludovico de Almeida e ao Professor Fabrício Casarejos, pelo apoio e estímulo que sempre me deram nos momentos mais difíceis mostraram sempre uma grande compreensão e solidariedade.

A fase de pesquisa de campo para este trabalho não teria sido possível sem a ajuda do Professor Luis Felipe Guanaes Rego, coordenador da Agenda Ambiental PUC-Rio. Quero, por isso, agradecer-lhe pela sua disponibilidade e incansável vontade de me ajudar neste trabalho.

Ao Conselho Nacional de Desenvolvimento Científico e TecnológicoCNPq, pela bolsa de estudos concedida, proporcionando condições para a realização dessa pesquisa.

Um agradecimento especial a todos da equipe do Núcleo Interdisciplinar de Meio Ambiente da PUC-Rio e aos membros da Comissão para a Sustentabilidade do Campus, que colaboraram nas entrevistas. Sem eles, não teria sido possível chegar a importantes conclusões.

A todos os professores do Programa de Pós-graduação em Metrologia para Qualidade e Inovação (PósMQI) e em especial ao Professor Maurício Frota, pela oportunidade de desenvolvimento profissional e acadêmico.

Por último, quero agradecer à minha família, em especial a minha mãe, minha filha e ao meu marido, pelo apoio, incentivo e compreensão com que suportaram a minha ausência durante este trabalho. 


\section{Resumo}

Costa, Andrea Viviane de Oliveira. Almeida, Maria Fatima Ludovico de. Casarejos, Fabrício (Orientadores). Indicadores de sustentabilidade para instituições de ensino superior: contribuições para a Agenda Ambiental PUC-Rio. Rio de Janeiro, 2012. 126 p. Dissertação de Mestrado - Programa de Pós-Graduação em Metrologia. Área de concentração: Metrologia para Qualidade e Inovação, Pontifícia Universidade Católica do Rio de Janeiro.

O objetivo da dissertação é propor um conjunto de indicadores de resultado e respectivas métricas a serem considerados pela PUC-Rio para mensurar a sustentabilidade do campus, em alinhamento às diretrizes da Agenda Ambiental PUC-Rio e a referenciais internacionais. No contexto institucional no qual a pesquisa se insere, considera-se que esse esforço propiciará ao NIMA/PUC-Rio, ao Grupo de Pesquisa Universidade Sustentável da PUC-Rio e aos diversos atores envolvidos na implementação das diretrizes e metas da Agenda o ferramental adequado para a mensuração da sustentabilidade da Universidade. A metodologia adotada compreende: (i) pesquisa bibliográfica e documental sobre instituições de ensino superior sustentáveis; metodologia de construção de indicadores, modelos de avaliação da sustentabilidade de IES e referenciais normativos em nível internacional; (ii) proposição de um modelo conceitual, base para a definição dos indicadores de resultado que expressem a sustentabilidade da PUC-Rio; (iii) elaboração de estudo de caso, tendo como foco a Agenda Ambiental PUC-Rio; (v) formulação das conclusões e recomendações para as entidades envolvidas e interessadas nos resultados da pesquisa. Destacam-se como resultados: (i) um modelo conceitual para avaliação da sustentabilidade de instituições de ensino superior, adaptado à realidade da PUC-Rio; e (ii) um conjunto consistente de indicadores de resultados para mensuração da sustentabilidade, visando a efetiva implementação da Agenda Ambiental, a melhoria contínua desse processo de mudança e a divulgação do desempenho sustentável da Universidade.

\section{Palavras-chave}

Metrologia; sustentabilidade; desenvolvimento sustentável; instituições de ensino superior; IES; campus sustentável; indicadores de sustentabilidade; Agenda Ambiental PUC-Rio. 


\section{Abstract}

Costa, Andrea Viviane de Oliveira. Almeida, Maria Fatima Ludovico de. Casarejos, Fabrício (Advisors). Sustainability indicators for higher education institutions: contributions to Environmental Agenda of PUCRio. Rio de Janeiro, 2012. 126 p. MSc. Dissertation - Programa de Pósgraduação em Metrologia. Concentration Area: Metrology for Quality and Innovation, Pontifícia Universidade Católica do Rio de Janeiro.

The objective of this dissertation is to propose a set of sustainability indicators for higher education institutions, within the perspective of application in the PUC-Rio, particularly for the evaluation of its Environmental Agenda initiative. Within the institutional context in which this research is situated, it is assumed that this effort will provide the proper tools to measure the University sustainability, focusing on the seven items of its Environmental Agenda. The research methodology encompasses: (i) bibliographical and documental review on sustainable higher education institutions (HEI), sustainability assessment tools and indicators for sustainable HEI and normative referential at international level; (ii) design of a conceptual model as a basis for building properly sustainability indicators, within the perspective of future application in PUC-Rio; (iii) development of a case study focusing on the PUC-Rio's Environmental Agenda; and (v) formulation of conclusions and recommendations for entities involved and interested in promoting sustainable higher education institutions, particularly those from PUC-Rio. The main results can be summarized as follows: (i) a conceptual model for evaluating sustainability in PUC-Rio; and (ii) a consistent set of sustainability indicators for measuring the results of implementation of the PUC-Rio’s Environmental Agenda.

\section{Keywords}

Metrology; sustainability; sustainable development; higher education institutions; HEI; sustainable campus; sustainability indicators; PUC-Rio's Environmental Agenda. 


\section{Sumário}

1. Introdução...................................................................................... 11

1.1 Definição do problema de pesquisa................................................. 15

1.2 Objetivos: geral e específicos.......................................................... 16

1.3 Motivação................................................................................... 17

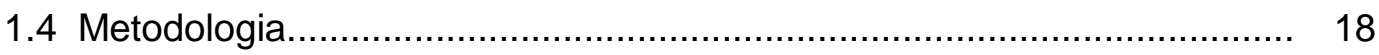

1.4.1 Fase exploratória e descrtitiva.................................................... 19

1.4.2 Pesquisa aplicada.......................................................................... 19

1.4.3 Fase conclusivo-propostiiva........................................................ 20

1.5 Estrutura da dissertação............................................................. 20

2. Desenvolvimento sustentável e o papel das instituições de ensino superior................................................................................................ 23

2.1 Conceitos básicos....................................................................... 25

2.1.1 Instituição de ensino superior sustentável......................................... 25

2.1.2 Sustentabilidade e sua avaliação..................................................... 28

2.2 Declarações e eventos para o alcance da sustentabilidade pelas IES.. 30

2.2.1 Declaração de Estocolomo.......................................................... 31

2.2.2 Declaração de Tbilisi......................................................................... 32

2.2.3 Declaração de Talloires................................................................. 32

2.2.4 Declaração de Halifax.................................................................... 34

2.2.5 Declaração de Quioto...................................................................... 35

2.2.6 Declaração de Swansea................................................................ 36

2.2.7 Carta de Copernicus...................................................................... 37

2.2.8 Declaração de Thessaloniki....................................... 38

2.2.9 Declaração Mundial sobre o Ensino Superior para o século XXI: Visão

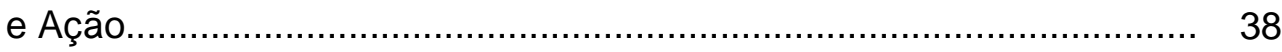

2.2.10 Declaração de Lüneburg......................................... 39

2.2.11 Declaração de Ubuntu................................................................. 40

2.3 Considerações finais sobre o capítulo................................................. 41

3. Modelos e ferramentas para quantificação da sustentabilidade de instituições de ensino superior...................................................... 42

3.1 Modelos e ferramentas gerais aplicáveis.............................................. 42

3.1.1. Global Reporting Initiative (GRI).................................................. 43

3.1.2 Norma ABNT NBR ISO 14001..................................................... 45

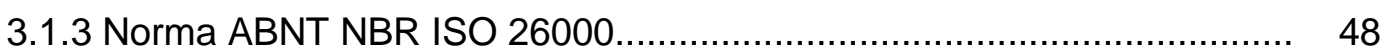

3.2 Modelos e ferramentas específicas para IES......................................... 49

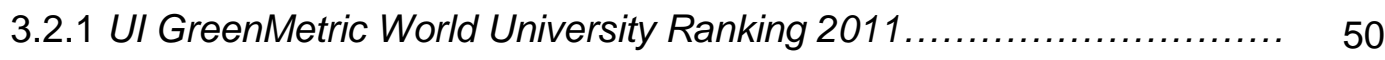


3.2.2 The College Sustainability Report Card $2011 \ldots \ldots \ldots \ldots \ldots \ldots \ldots \ldots \ldots . . \ldots \ldots$

3.2.3 Good Company's Sustainable Pathways Toolkit....................... 51

3.2.4 National Wildlife Federation's State of the Campus Environment...... 52

3.2.5 ULSF's Sustainability Assessment Questionnaire ...................... 52

3.2.6 Campus Consortium for Environmental Excellence (C2E2)............ 53

3.2.7 Auditing Instrument for Sustainable Higher Education........................ 54

3.2.8 Campus Sustainability Assessment Framework...................... 54

3.3 Considerações finais sobre o capítulo.................................................. 55

4. Modelo conceitual para mensuração da sustentabilidade de instituições de ensino superior..

4.1 Visão geral do modelo conceitual para mensuração da sustentabilidade. 57

4.1.1 Características do modelo................................................................ 58

4.1.2 Representação gráfica do modelo................................................... 59

4.2. Visão modular......................................................................... 60

4.2.1 Módulo 1: Biodiversidade............................................................... 61

4.2.2 Módulo 2: Água......................................................................... 62

4.2.3 Módulo 3: Energia...................................................................... 64

4.2.4 Módulo 4: Atmosfera........................................................................ 66

4.2.5 Módulo 5: Materiais.................................................................... 67

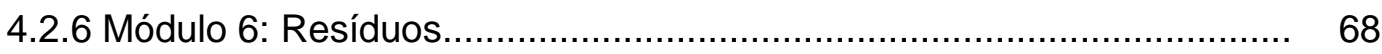

4.2.7 Módulo 7: Educação ambiental...................................................... 69

4.3 Sistema de mensuração: definição dos indicadores por módulo........... 71

4.3.1 Conceitos básicos para construção de indicadores.............................. 71

4.3.2 Taxonomia de indicadores........................................................... 73

4.3.3 Construção de indicadores............................................................ 73

4.4 Considerações finais sobre o capítulo.................................................. 83

5. Estudo de caso: contribuição para a Agenda Ambiental PUC-Rio.... 84

5.1 Questões do estudo de caso............................................................... 85

5.2 Tipo de caso: por que estudo de caso único holístico?........................... 85

5.3 Unidade de análise: Agenda Ambiental PUC-Rio................................ 86

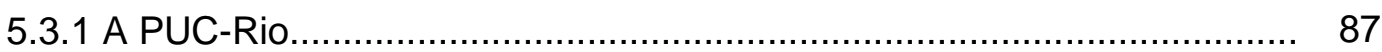

5.3.2 O Núcleo Interdisciplinar de Meio Ambiente - NIMA........................... 88

5.3.3 A Agenda Ambiental PUC-Rio......................................................... 89

5.4 Proposição de indicadores de resultado por eixo temático.................... 90

5.4.1 Indicadores 'candidatos' representativos das diretrizes da Agenda..... 91

5.4.2 Instrumento para seleção e priorização dos indicadores 'candidatos'.... 94

5.5 Considerações finais sobre o estudo de caso........................................ 96

6. Conclusões e recomendações........................................................... 97

Referências bibliográficas.................................................................... 102

Anexo 1 - Indicadores de sustentabilidade de IES - Partes 1 e 2............ 113

Anexo 2 - Instrumento para seleção e priorização de indicadores.......... 118 


\section{Lista de Figuras}

Figura 1.1 - Desenho da pesquisa, seus componentes e métodos................. 18

Figura 2.1 - Papel das IES na sociedade..................................................... 24

Figura 2.2 - Principais fluxos de um campus universitário.............................. 26

Figura 4.1 - Modelo conceitual para mensuração da sustentabilidade de IES.. 59

Figura 4.2 - Nível de agregação de dados e indicadores............................... 72

Figura 4.3 - Fluxograma de construção de indicadores em 8 passos......... $\quad 74$

Figura 4.4 - Exemplo didático de uma matriz de avaliação quantitativa de $\quad 80$ indicadores

Figura 5.1 - Seleção do estudo de caso, segundo Yin (2005)........................ 85

\section{Lista de Quadros}

Quadro 2.1 - Declarações e eventos relevantes pra a implementação de IES sustentáveis.

Quadro 3.1 - Modelos e ferramentas gerais aplicáveis às IES........................ 42

Quadro 3.2 - Definição dos temas da Norma ABNT NBR ISO 26000............... 48

Quadro 4.1 - Exemplos de critérios para seleção de indicadores..................... 76

Quadro 5.1 - Proposta de indicadores de resultado para avaliação estratégica da Agenda PUC-Rio....................................................... 92

Quadro 5.2 - Especialistas indicados para a fase de seleção e priorização de indicadores 'candidatos'.... 


\section{Lista de Siglas}

AASHE- The Association for the Advancement of Sustainability in Higher Education

ACU - The Association of Commonwealth Universities

CSD - The Commission on Sustainable Development of the United Nations

CRE - Conference of European Rectors

DESD - Decade of Education for Sustainable Development

EFQM - European Foundation for Quality Management

EUA - The European University Association

INK - Instituut Nederlandse Kwaliteit

GEE - Gases de Efeito Estufa

GRI - Global Reporting Initiative

IAU - The International Association of Universities

IES - Instituição de Ensino Superior

ISO - International Organization for Standardization

IUCN - International Union for Conservation of Nature

NIMA/PUC-Rio - Núcleo Interdisciplinar de Meio Ambiente da Pontifícia

Universidade Católica do Rio de Janeiro

P\&D - Pesquisa e Desenvolvimento

PUC-Rio - Pontifícia Universidade Católica do Rio de Janeiro

SGA - Sistema de Gestão Ambiental

TEP - Tonelada Equivalente de Petróleo

UI - Universitas Indonesia

ULSF - University Leaders for a Sustainable Future

UNCED - United Nations Conference on Environment and Development

UNEP - United Nations Environment Programme

UNESCO - United Nations Educational, Scientific and Cultural Organization

UNU - The United Nations University

WCED - World Commission on Environment and Development

WSSD - The World Summit for Sustainable Development 